\title{
Substrate dependence of large ordinary magnetoresistance in sputtered Bi films
}

\author{
Jen-Hwa Hsu ${ }^{\mathrm{a}, \mathrm{b}, *}$, Yung-Shin Sun ${ }^{\mathrm{b}}$, Hong-Xian Wang ${ }^{\mathrm{c}}$, P.C. Kuo ${ }^{\mathrm{b}, \mathrm{c}}$, \\ Tsung-Hsien Hsieh ${ }^{\mathrm{a}}$, Chi-Te Liang ${ }^{\mathrm{a}}$ \\ ${ }^{a}$ Spintronics Laboratory, Department of Physics, National Taiwan University, Taipei 106, Taiwan \\ ${ }^{\mathrm{b}}$ Center for Nanostorage Research, National Taiwan University, Taipei 106, Taiwan \\ ${ }^{\mathrm{c}}$ Department of Material Engineering, National Taiwan University, Taipei 106, Taiwan
}

\begin{abstract}
The Bi films of different crystalline orientations can be obtained from growing on selected substrates such as $\operatorname{Si}(100)$,

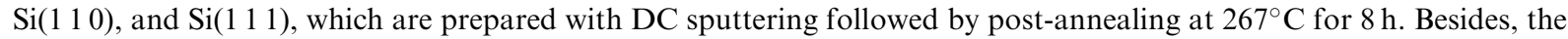
positive large ordinary magnetoresistance effect is shown to be highly anisotropic. We have also observed distinct oscillation periods of Shubnikov de-Haas oscillations at low temperatures. This is due to the different orientation of the cyclotron orbits with respect to the films.
\end{abstract}

(C) 2004 Elsevier B.V. All rights reserved.

PACS: 71.55.Ak; 73.50.Jt; 81.15.Cd; 72.15.Lh

Keywords: Magnetoresistance; Bismuth; Shubnikov de-Haas oscillation; Sputtering

Bismuth, a semi-metal material, has been studied extensively in the last century because Bi possesses many unique electronic properties [1]. With these unusual characteristics, the $\mathrm{Bi}$ thin films have been used to investigate the classical as well as quantum finite size effect and localization behavior. Besides, the Shubnikov-de Haas $(\mathrm{S}-\mathrm{dH})$ oscillations due to the Landau quantization of the cyclotron orbits of the carriers was also first discovered in $\mathrm{Bi}$ single crystal at low temperatures [2]. Recently, the research of Bi has been extended into bismuth nanowires that provide an excellent system to investigate the quantum confinement effect on the electronic properties of quasi-1D systems; for example see Ref. [3]. In the near future, nanostructured bismuth is expected to play a important role in spintronics, e.g., the interconnect between the spin memory cells.

*Corresponding author.Department of physics, National Taiwan University, 1 Roosevelt R., Sec.4, Tapei 106, Taiwan. Tel.: 886-2-23661867; fax: 886-2-33665167.

E-mail address: jhhsu@phys.ntu.edu.tw (J.-H. Hsu).
Another issue related to Bi films is the ordinary magnetoresistance (OMR). The Lorentz force in the magnetic field causing helical motion of charge carriers has been realized responsible for OMR effect. The $\omega_{\mathrm{c}} \tau$ value is found to act as the dominant factor to determine the MR value for the OMR effect, where $\omega_{\mathrm{c}}=e H / \mathrm{m}^{*} c$ is the cyclotron frequency, $\tau$ is the carrier relaxation time, and $m *$ is the effective mass of the carrier. It is straightforward to show that $\omega_{\mathrm{c}} \tau=H \sigma /$ nec. In the previous expression $\sigma=n e^{2} \tau / m *$ is the conductivity and $n$ is the carrier concentration. In the metals such as $\mathrm{Cu}$, $\mathrm{Ag}$, and $\mathrm{Au}$, the dependence of $\omega_{\mathrm{c}} \tau$ on magnetic field can be approximately described by $\omega_{\mathrm{c}} \tau \fallingdotseq 0.005 H$, where $H$ is the magnetic field in unit of Tesla. Thus, their OMR value is smaller than $1 \%$ under $1 \mathrm{~T}$. To have a substantial MR, $\omega_{\mathrm{c}} \tau$ should be at least of order 1. It has been found that the $\omega_{\mathrm{c}} \tau$ value can be increased up to 100 times for Bi from those for metals. So the Bi film has potential to exhibit large OMR. In the past, it has been understood that only single-crystal Bi films can give large OMR effect. Bi films made by traditional vapor deposition methods, such as sputtering and evaporation, 


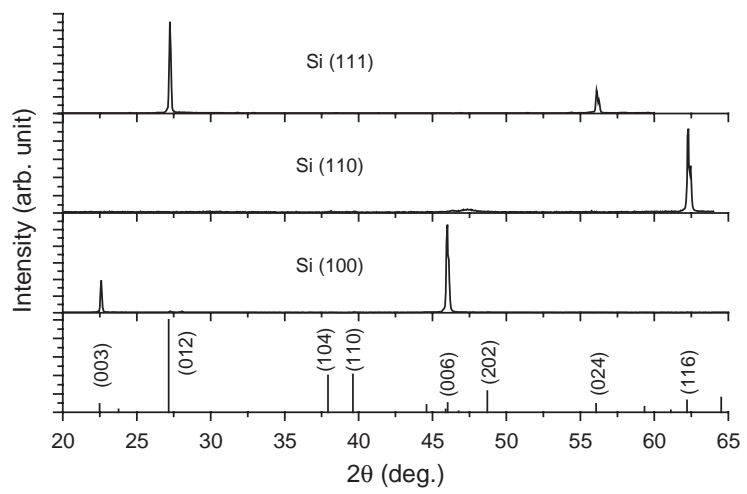

Fig. 1. $\theta 2 \theta \mathrm{X}$-ray diffraction patterns for Bi films with different substrates. The diffraction peaks for powder $\mathrm{Bi}$ are also included for comparison.

are polycrystalline with very small grains, e.g. $10 \mathrm{~nm}$ large, which often exhibit disappointingly small effect. In order to grow Bi films with large MR, more sophisticated processes such as molecular-beam-epitaxy were used. In this work, however, we report an extremely large OMR effect at room temperature for Bi films on Si wafer fabricated with DC sputtering followed by a post-annealing treatment. Our sputtered Bi films reveal many unique properties which show crystalline orientation dependence.

The Bi films were made by DC magnetron sputtering using a current of only $20 \mathrm{~mA}$. After as-deposited films were removed from the sputter, the annealing step was undertaken immediately. The annealing temperature was set at $267^{\circ} \mathrm{C}, 4^{\circ} \mathrm{C}$ below the melting point of $\mathrm{Bi}$. And the annealing time is $8 \mathrm{~h}$. The film structure and morphology were investigated by X-ray diffraction and transmission electron microscopy (TEM), respectively. $\mathrm{MR}$ is defined to be $\mathrm{MR}=\{R(\mathrm{H})-R(0)\} / R(0)$.

In Fig. 1, the representative X-ray diffraction patterns of Bi films with different substrate orientations of $\mathrm{Si}$ wafer are shown. It has been shown that the substrate indeed plays an important role for expitaxy. For example, the Bi films with $\mathrm{Si}(100)$ substrate prefer to grow along [0 03 3] direction. On the other hand, the film structure with $\mathrm{Si}\left(\begin{array}{lll}1 & 1 & 1\end{array}\right)$ develops into a large grained [0 $\left.\begin{array}{ll}0 & 1\end{array}\right]$ texture. From TEM pictures, the shape of bismuth grains is about spherical and their size is $130 \mathrm{~nm}$ in

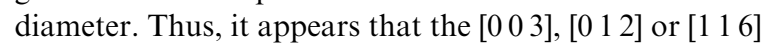
preferred axes of $\mathrm{Bi}$ aligns perpendicular to the substrate, but the in-plane structure of the film would be slightly twined.

The zero-field, room-temperature resistivity of a single-crystal $\mathrm{Bi}$ film is about $1.15 \mu \Omega \mathrm{m}$ at $300 \mathrm{~K}$, while the resistivity of a polycrystalline Bi film is in general larger than $4.25 \mu \Omega \mathrm{m}$ depending on film quality. Our data of high-temperature annealed films fall into the

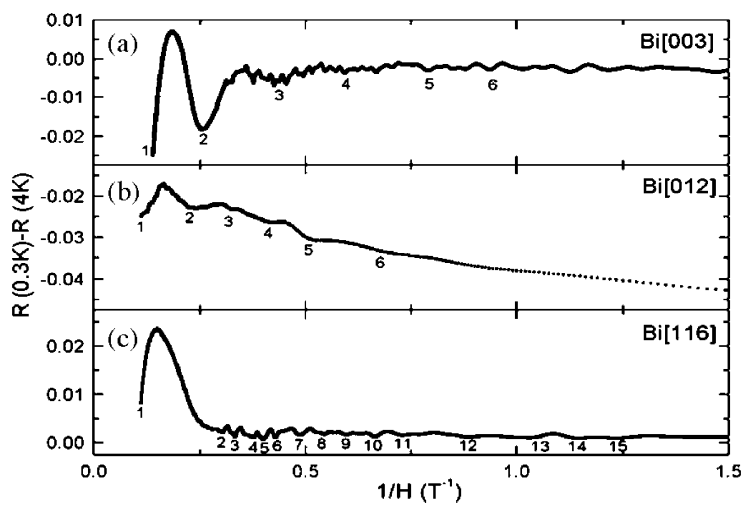

Fig. 2. Shubnikov-de Haas oscillations versus inverse magnetic field $1 / H$ for three orientations.

range of $1.5-2 \mu \Omega \mathrm{m}$, indicating the formation of Bi films with good crystallinity and low defects. The MR curves shows no hysteresis. And the MR value is more than $300 \%$ under $5 \mathrm{~T}$ for $\mathrm{Si}\left(\begin{array}{ll}1 & 1\end{array}\right)$. Compared with published data from other's works [4], to our knowledge, this is the highest room temperature MR so far reported for $\mathrm{Bi}$ films. Besides, the MR has crystalline anisotropic behavior. $\mathrm{Bi}\left[\begin{array}{ll}0 & 12\end{array}\right]$ has the largest $\mathrm{MR}$ whereas it is reduced to $150 \%$ for $\mathrm{Bi}[003]$. It is completely due to highly anisotropic Fermi surface of Bi.

The $\mathrm{S}-\mathrm{dH}$ oscillations with $\mathbf{H}$ applied perpendicular to the film plane for three different crystalline orientations are shown in Fig. 2 as a funcion of $1 / H$. In order to remove the background, the MR values at $4 \mathrm{~K}$ have been substracted. The minima in the curves are considered as the locations of the $\mathrm{S}-\mathrm{dH}$ oscillations. It is noted that the oscillations are almost periodic in $1 / H$ for [0 03 3] and [0 12 ] oriented films. The order $n$ of the oscillations depends essentially linearly on the inverse field. However, due to the highly non-symmetric characteristic along [ $\left.1 \begin{array}{lll}1 & 1\end{array}\right]$ orientation, the oscillations of multiple periods are expected to be observed as indicated in Fig. 2. The detailed quantitative analysis will be given in a separate paper.

In summary, we have demonstrated that a large MR effect of Bi films can be grown from sputtering subjected to a subsequent suitable post-annealing procedure below the melting point of $\mathrm{Bi}$. The enhancement is $300 \%$ under $5 \mathrm{~T}$ at room temperature. Furthermore, the MR and $\mathrm{S}_{-}$ $\mathrm{dH}$ oscillation are found to be oriented dependent.

\section{References}

[1] G.E. Smith, G.A. Baraff, J.M. Rowell, Phys. Rev. 135 (1964) A1118. 
[2] L.W. Shubnikov, W.J. de Haas, Proc. Acad. Sci. Amsterdam 33 (1930) 130, 363, 418.

[3] J.L. Costa-Kramer, N. Garcia, H. Olin, Phys. Rev. Lett. 78 (1997) 4990;;

K. Liu, C.L. Chien, P.C. Searson, Phys. Rev. B 58 (1998) R14681.
[4] F.Y. Yang, K. Liu, K. Hong, D.H. Reich, P.C. Searson, C.L. Chien, Science 284 (1999) 1335;

F.Y. Yang, Kai Liu, C.L. Chien, Searson, Phys. Rev. Lett. 82 (1999) 3328;

Sunglae Cho, et al., Appl. Phys. Lett. 79 (2001) 3651. 